\title{
The effect of oxybuprocaine on corneal thickness as measured with optical coherence tomography
}

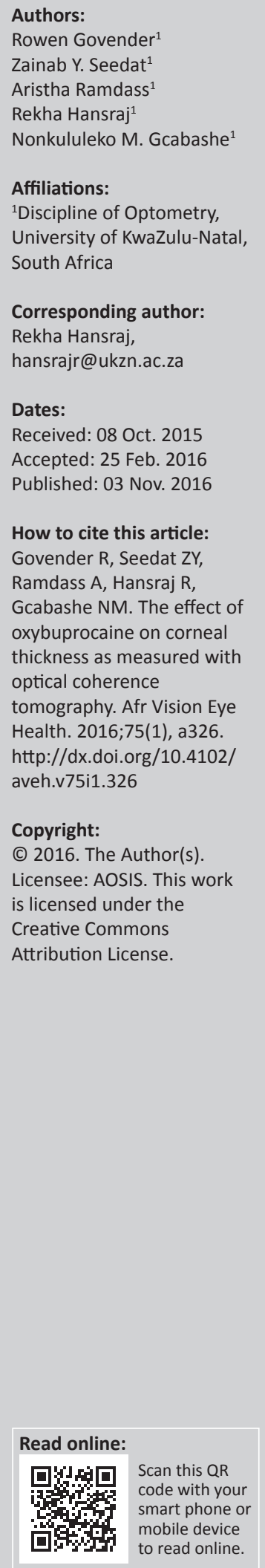

Authors:

Rowen Govender

Affiliations:

${ }^{1}$ Discipline of Optometry,

University of KwaZulu-Natal,

Rekha Hansraj,

Dates:

Accepted: 25 Feb. 2016

How to cite this article:

Govender R, Seedat ZY,

Gcabashe NM. The effect of

oxybuprocaine on corneal

thickness as measured with

4102

aveh.v75i1.326

Copyright:

is licensed under the

Creative Commons

mobile device

to read online.
Background: No previous study in South Africa has used optical coherence tomography $(\mathrm{OCT})$ in assessing corneal thickness changes following the instillation of an anaesthetic.

Aim: The purpose of this study was to investigate the short-term effects of oxybuprocaine $0.4 \%$ on corneal thickness.

Setting: The study was conducted at a university eye clinic in Westville, KwaZulu-Natal.

Methods: A total of $34(N=34)$ participants, of both genders, aged 18-25 years participated in the study. Data collection commenced once ethical clearance was obtained. Baseline measurements of central corneal thickness (CCT) (within $0 \mathrm{~mm}-2 \mathrm{~mm}$ of the corneal apex) and mid-peripheral corneal thickness (MPCT) (within $2 \mathrm{~mm}-5 \mathrm{~mm}$ of the corneal apex) were taken using the Optovue iVue 100 OCT. Measurements were obtained using the standard iVue 100 corneal pachymetry map. One drop of oxybuprocaine was then instilled using a sterile technique in the right eye (RE) of each participant. Central and MPCT measurements were obtained using the OCT at time intervals of $30 \mathrm{~s}$ for the first $2 \mathrm{~min}$ and then for $60-\mathrm{s}$ intervals up until $5 \mathrm{~min}$. Baseline central and MPCT were then measured for the left eye (LE) using the OCT. The effect of two drops of oxybuprocaine on CCT was also investigated on the left eye only using the same procedure as on the right eye.

Results: There were minimal fluctuations of the CCT and MPCT in the $5 \mathrm{~min}$ following instillation of either one or two drops of oxybuprocaine. The fluctuation of both the CCT and MPCT was within $10 \mu \mathrm{m}$ irrespective of dosage and returned to baseline thickness after $5 \mathrm{~min}$.

Conclusion: Clinically, short-term CCT and MPCT changes following the instillation of oxybuprocaine are not large enough to affect the accuracy of Goldmann applanation tonometry.

\section{Introduction}

The cornea serves not only as the primary refractive surface of the eye $\mathrm{e}^{1}$ but also as a surface against which intraocular pressure measurements (IOP) can be taken, among other things. Accurate IOP measurements are critical in the early detection, management and hence prognosis of glaucoma. Goldmann applanation tonometry (GAT) is widely accepted as the international gold standard for direct contact IOP measurements. ${ }^{2}$ The accuracy of GAT measurements depends on many factors including corneal thickness, curvature and axial length. ${ }^{3}$ It has been shown that an accurate reading of IOP using GAT is obtained with a corneal thickness of about $0.52 \mathrm{~mm} .{ }^{4}$ Furthermore, thicker corneas lead to an overestimation of IOP and thinner corneas to an underestimation of IOP. ${ }^{4,5,6,7}$ Central corneal thickness (CCT) has been shown to have a substantial effect on IOP readings obtained by $\mathrm{GAT}^{8,9,10}$, with previous studies demonstrating a positive correlation between CCT and IOP..$^{11,12}$

When measuring the IOP of a patient with GAT, a local anaesthetic is used to anaesthetise the cornea, ensuring minimal discomfort to the patient. Corneal thickness changes following instillation of an anaesthetic occur and have been attributed to the preservatives causing damage to the corneal epithelium and endothelium, thereby altering endothelial metabolism and disrupting the Na-K endothelial pump resulting in corneal oedema. . $^{13,14,15}$

Previous studies into this change in corneal thickness have produced varying results. Some studies ${ }^{13,16}$ have shown varying corneal thickness over a period of 10 min while others ${ }^{14,15,17,18}$ have found no significant changes following instillation of an anaesthetic. Variation in the results reported by previous studies ${ }^{13,14,15,17,18}$ may be attributed to different methods of measuring corneal thickness which have included ultrasound pachymetry, topography with an Orbscan, non-contact specular microscopy or the rotating Scheimpflug camera (Pentacam). 
One of the challenges of ultrasound pachymetry is that it is a contact method requiring the use of an anaesthetic. ${ }^{13,19}$ Ogbuehi et al..$^{15}$ had therefore asserted that the effect of local anaesthetics on corneal thickness as measured by different pachymetry devices remains unclear. Only one study ${ }^{19}$ used non-contact optical pachymetry but this study was different from the others in that it evaluated corneal thickness changes following instillation of a combination of $0.5 \%$ proparacaine and $0.25 \%$ sodium fluorescein. No previous study in South Africa has used optical coherence tomography (OCT) in assessing corneal thickness changes following the instillation of just an anaesthetic. This is important as comparative studies ${ }^{20,21}$ have concluded that there was a significant difference in thickness measurements produced by ultrasound pachymetry compared with OCT, with ultrasound pachymetry overestimating corneal thickness slightly.

The method of OCT is advantageous as it allows an assessment of corneal thickness without the use of an anaesthetic, and Asensio et al. ${ }^{17}$ recommended that these instruments should be used by future studies to investigate corneal epithelial thickness changes following instillation of an anaesthetic. OCT has also been found to have a high degree of repeatability and reproducibility ${ }^{22}$ and would thus be useful in such studies.

Many of the previous studies ${ }^{13,15,18}$ have used only one drop of anaesthetic while others ${ }^{14,17}$ studied the effect of only two drops of anaesthetic. Only one study ${ }^{16}$ compared the differences between one and two drops of anaesthetic on corneal thickness over time but with optical pachymetry. Clinicians may vary in their preference of one or two drops of anaesthetic for various procedures without necessarily considering the side-effects of dosage changes. Previous studies ${ }^{13,14,15,18}$ have also only concentrated on the CCT. Only studies by Asensio et al. ${ }^{17}$ and Mukhopadhyay et al. ${ }^{19}$ investigated the effect on mid-peripheral corneal thickness (MPCT) as well, which becomes relevant when considering refractive surgery for example. The current study therefore set out to investigate the changes in central and midperipheral corneal thickness following instillation of one or two drops of oxybuprocaine as measured by OCT.

\section{Research methods and design Study design}

The study employed a cross-sectional, descriptive design. The study population consisted of undergraduate students aged between 18 and 25 years based at the UKZN Westville campus. The convenience sampling method was employed to select 34 participants, of either gender. Most participants were either Black or Indian South Africans.

\section{Setting}

The study was conducted at the optometry clinic of the University of KwaZulu-Natal, Westville campus.

\section{Data collection}

Preliminary tests were carried out on each participant, including a brief ocular and medical history, autorefraction and ophthalmoscopy, to assess internal health and slit lamp biomicroscopy to rule out infections and abnormalities of the external surfaces of the eye. The exclusion criteria included past or current history of ocular pathology and anomalies, the use of systemic or ocular medication, contact lens wearers and allergy to oxybuprocaine. An initial Optovue OCT measurement of central (within $0 \mathrm{~mm}-2 \mathrm{~mm}$ of the corneal apex) and mid-peripheral (within $2 \mathrm{~mm}-5 \mathrm{~mm}$ of the corneal apex) corneal thickness of the right eye was taken using the Optovue iVue optical coherence tomographer. Measurements were obtained using the standard iVue 100 corneal pachymetry map. One drop of oxybuprocaine HCL $0.4 \%$ was then instilled using a sterile technique into the right eye. Upon instillation, the punctum was occluded using the index finger and a tissue for $10 \mathrm{~s}$, the timer was then started $15 \mathrm{~s}$ after the instillation as oxybuprocaine has an activating time of approximately $15 \mathrm{~s}$. A dropper was used to measure out a single drop with the subject's head in a supine position to ensure maximum absorption.

The corneal thickness of the right eye of the participant was then measured using the OCT, and recorded, at various time intervals of $30 \mathrm{~s}$ for the first $2 \mathrm{~min}$ and then 60 -s intervals up to $5 \mathrm{~min}$ post-instillation. Thereafter, a new baseline corneal thickness (central and mid-peripheral) was measured on the left eye of the same participant. The effect of two drops of oxybuprocaine on corneal thickness was then investigated on the left eye using the same procedure as outlined above. The same process was repeated in all participants with the readings being taken in the afternoons only. To ensure uniformity and standardisation, one researcher was responsible for all the OCT measurements.

\section{Data analysis}

The results of all tests were captured and analysed using the Statistical Package for Social Sciences (SPSS v.21) and Microsoft Excel 2010, under the guidance of the faculty statistician. The paired $t$-test and the linear mixed-effects model tests were used to analyse the results.

\section{Ethical considerations}

Ethical clearance was obtained from the Biomedical Research and Ethics Committee of the University of KwaZulu-Natal (ethical clearance no: SHSEC 041/13). The participants gave written informed consent for this research study. The relevant tenets of the Declaration of Helsinki were adhered to throughout this study.

\section{Results}

The mean CCT and standard deviation, at baseline, for the right eyes were $494.76 \mu \mathrm{m} \pm 34.48 \mu \mathrm{m}$ and that for the left eyes were $496.47 \mu \mathrm{m} \pm 35.89 \mu \mathrm{m}$. Using the paired $t$-test no significant difference at a $95 \%$ level of confidence was found 
between the baseline CCT readings for the right and left eyes $(p>0.05)$. Figure 1 was plotted using the mean deviation from baseline CCT measurements at the various time intervals up to $300 \mathrm{~s}$. Following instillation of one drop of oxybuprocaine HCL $0.4 \%$, the CCT was observed to increase (above $250 \mu \mathrm{m}$ ) from the mean deviation of the baseline readings within the first $30 \mathrm{~s}$. This increase was found to be statistically significant using the paired $t$-test $(p=0.006)$. Clinically, the mean increase is small. After $30 \mathrm{~s}$, there was a gradual decrease in mean deviation from baseline CCT at

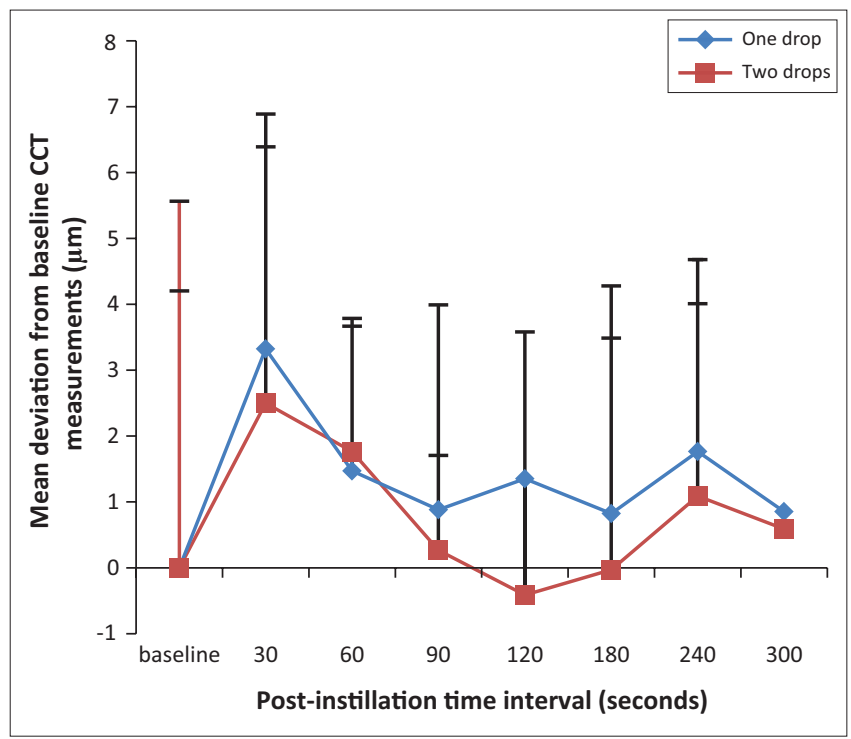

FIGURE 1: Variation in mean central corneal thickness measured by Optovue optical coherence tomography over a period of $300 \mathrm{~s}$ after instillation of one drop of oxybuprocaine $\mathrm{HCL} 0.4 \%$ in the right eye (blue dotted line) and two drops of oxybuprocaine HCL $0.4 \%$ in the left eye (red dashed line) of 34 participants aged $18-25$ years. The arrow bars represent $95 \%$ limits of confidence intervals. regular intervals between 60 and $300 \mathrm{~s}$ ( $5 \mathrm{~min}$ ) as the mean deviation returned to the baseline measurements. There was no significant difference $(p>0.05)$ between baseline and any other interval up to $300 \mathrm{~s}$.

Variation in CCT following instillation of two drops of oxybuprocaine HCL $0.4 \%$ showed a similar trend to that of one drop only. There was a significant increase in CCT compared with baseline at $30 \mathrm{~s}$ (paired $t$-test; $p=0.007$ ) after which there was a decrease in CCT reaching baseline at approximately $100 \mathrm{~s}$. Thereafter there was a slight decrease in corneal thickness below baseline and a return to baseline at $180 \mathrm{~s}$, after which an increase was observed again. The changes after $30 \mathrm{~s}$, however, were insignificant when compared with baseline readings (paired $t$-test; $p>0.05$ ). Thus, the greatest deviation in CCT from baseline occurred at $30 \mathrm{~s}$ following the instillation of either one or two drops of oxybuprocaine HCL $0.4 \%$.

In terms of MPCT, the superior quadrant (see Table 1) was the thickest in both the right and left eyes (mean corneal thickness of $561.27 \mu \mathrm{m}$ and $561.45 \mu \mathrm{m}$, respectively) prior to instillation of oxybuprocaine HCL $0.4 \%$. At baseline, the thinnest mean corneal thicknesses were found in the temporal quadrant $(522.25 \mu \mathrm{m})$ for the right eyes and the nasal quadrant $(527.35 \mu \mathrm{m})$ for the left eyes.

Following instillation of either one or two drops of oxybuprocaine HCL $0.4 \%$, increases or decreases in MPCTs were recorded. The linear mixed models were used in all the analyses of variation of mean MPCTs. The mean differences for intervals $60,90,120,180,240$ and $300 \mathrm{~s}$ were compared with that of $30 \mathrm{~s}$. The $t$-values of the results for the linear mixed models analyses are shown in Table 2.

TABLE 1: Variation in mid-peripheral mean corneal thickness measured by Optovue optical coherence tomography over a period of $300 \mathrm{~s}$ after instillation of one drop of oxybuprocaine $\mathrm{HCL} 0.4 \%$ in the right eye and two drops of oxybuprocaine $\mathrm{HCL} 0.4 \%$ in the left eye of 34 participants, aged $18-25$ years.

\begin{tabular}{|c|c|c|c|c|c|c|c|c|}
\hline \multirow{3}{*}{$\begin{array}{l}\text { Post-instillation } \\
\text { time (seconds) }\end{array}$} & \multicolumn{8}{|c|}{ Mean corneal thickness measurements of the mid-peripheral quadrants $(\mu \mathrm{m})$} \\
\hline & \multicolumn{2}{|c|}{ Superior } & \multicolumn{2}{|c|}{ Nasal } & \multicolumn{2}{|c|}{ Inferior } & \multicolumn{2}{|c|}{ Temporal } \\
\hline & One drop & Two drops & One drop & Two drops & One drop & Two drops & One drop & Two drops \\
\hline 0 & 561.27 & 561.45 & 552.73 & 527.35 & 533.63 & 536 & 522.25 & 551.98 \\
\hline 30 & 561.71 & 569.92 & 558.57 & 525.82 & 538.78 & 534 & 524.45 & 551.75 \\
\hline 60 & 566.35 & 571.35 & 554.86 & 528.22 & 533.04 & 534.9 & 526.75 & 554.53 \\
\hline 90 & 565.39 & 568.57 & 541.39 & 523.25 & 536.29 & 532.94 & 524.16 & 550.65 \\
\hline 120 & 560.63 & 566.9 & 555.14 & 524.31 & 531.2 & 530.82 & 522.1 & 550.61 \\
\hline 180 & 567.14 & 564.8 & 559.24 & 524.33 & 532.49 & 533.18 & 520.82 & 552.63 \\
\hline 240 & 569.14 & 566.25 & 553.41 & 521.06 & 528.76 & 533.43 & 523.02 & 553.31 \\
\hline 300 & 566.61 & 563.45 & 546.57 & 527.29 & 525.27 & 531.65 & 526.25 & 550.41 \\
\hline
\end{tabular}

TABLE 2: The $t$-values of the linear mixed models analyses on the mean corneal thickness differences (from baseline central corneal thickness) in the mid-peripheral quadrants measured by Optovue optical coherence tomography over a period of $300 \mathrm{~s}$ after instillation of one drop of oxybuprocaine HCL $0.4 \%$ in the right eye and two drops of oxybuprocaine $\mathrm{HCL} 0.4 \%$ in the left eye of 34 participants, aged $18-25$ years.

\begin{tabular}{|c|c|c|c|c|c|c|c|c|}
\hline \multirow[t]{3}{*}{ Time (seconds) } & \multicolumn{8}{|c|}{$t$-values of the linear mixed models analyses on the mean corneal thickness differences in mid-peripheral quadrants } \\
\hline & \multicolumn{2}{|c|}{ Superior } & \multicolumn{2}{|c|}{ Nasal } & \multicolumn{2}{|c|}{ Inferior } & \multicolumn{2}{|c|}{ Temporal } \\
\hline & One drop & Two drops & One drop & Two drops & One drop & Two drops & One drop & Two drops \\
\hline 30 & 0.081 & 1.304 & 1.535 & -1.430 & 2.143 & $-2.341 *$ & 0.712 & -0.671 \\
\hline 60 & 0.754 & -0.369 & -0.719 & 0.836 & $-1.880 *$ & 1.538 & 0.633 & 0.096 \\
\hline 90 & 0.598 & -0.578 & $-3.331^{*}$ & $2.003 *$ & -0.815 & 0.331 & -0.081 & -0.157 \\
\hline 120 & -0.175 & -0.223 & -0.665 & 0.263 & $-2.483^{*}$ & 1.021 & -0.650 & 0.237 \\
\hline 180 & 0.881 & -1.210 & 0.129 & -0.296 & $-2.059 *$ & 1.266 & -1.002 & 0.880 \\
\hline 240 & 1.205 & -1.273 & -1.000 & 0.054 & $-3.278^{*}$ & $2.186 *$ & -0.395 & 0.586 \\
\hline 300 & 0.795 & -1.304 & $-2.237^{*}$ & $1.847 *$ & $-4.420 *$ & $2.581 *$ & 0.498 & -0.612 \\
\hline
\end{tabular}

$*$, Significant at $90 \%$ confidence level. 
Significant differences in mean corneal thicknesses from baseline were observed in the inferior quadrant at all postinstillation intervals except for the 30- and 90-s intervals for one drop of oxybuprocaine HCL $0.4 \%$. Following the instillation of two drops of oxybuprocaine HCL $0.4 \%$, significant differences from baseline were also noted at the 30-, 240- and 300-s intervals in this quadrant. The only other quadrant that showed significant change from baseline following both one and two drops of oxybuprocaine HCL $0.4 \%$ were the nasal quadrants at the 90 - and 300 -s postinstillation intervals.

\section{Discussion}

Variation in CCT was observed following instillation of one drop of oxybuprocaine HCL $0.4 \%$ but this thickness difference was only statistically significant at the first $30 \mathrm{~s}$ postinstillation. CCT then returned to values that were not significantly different from baseline. A very similar trend was noted with instillation of two drops of oxybuprocaine HCL $0.4 \%$, with a significant increase compared with baseline in the first $30 \mathrm{~s}$ only. In both instances, it appears that corneal thickness returns to baseline after $300 \mathrm{~s}$ ( $5 \mathrm{~min}$ ). The increase in corneal thickness has been attributed to the diffusion of the topical anaesthetic into the deep stromal layers which causes an inhibition of the endothelial cell metabolism, resulting in corneal oedema and the subsequent increase in corneal thickness. ${ }^{13,15}$ In addition, Penne and Tabbara ${ }^{23}$ found that (for the epithelium of rabbits) the use of oxybuprocaine HCL $0.4 \%$ affected the $\mathrm{Na} / \mathrm{K}$ pump, resulting in an increased osmotic pressure, leading to an increased hydration of the stroma and subsequently increase in corneal thickness. Herse and Sui ${ }^{16}$ attributed the recovery to baseline CCT within 7-8 min to the exponential modelling of the corneal oedema recovery function. This was also observed in the current study as the corneal thickness appeared to be closest to baseline at around 5 min post-instillation. It is expected that this would be the physiological change with any local anaesthetic. Nam et al. ${ }^{13}$ compared corneal thickness changes of oxybuprocaine to that of proparacaine. Proparacaine increased CCT by $8.6 \mu \mathrm{m}$ compared with the $7.7 \mu \mathrm{m}$ increase with oxybuprocaine, but was followed by re-stabilisation after $80 \mathrm{~s}$.

Similar findings have been reported in previous studies. ${ }^{13,14,15,17}$ Rosa et al..$^{14}$ found no significant changes in corneal thickness $5 \mathrm{~min}$ after the instillation of oxybuprocaine $(0.4 \%)$ as measured with an Oculus Pentacam. A temporary increase in corneal thickness which returned to normal after about $8 \mathrm{~min}$ was noted by Herse and Siu. ${ }^{16}$ Ogbuehi et al. ${ }^{15}$ found no significant change in CCT up to $10 \mathrm{~min}$. Nam et al. ${ }^{13}$ reported a $7.7 \mu \mathrm{m}$ increase in corneal thickness and concluded that there was corneal thickness instability following the instillation of oxybuprocaine but that the CCT returned to baseline after $80 \mathrm{~s}$. Asensio et al. ${ }^{17}$ found no significant change in CCT with two drops of $0.4 \%$ oxybuprocaine $3 \mathrm{~min}$ postinstillation using Orbscan Topography System II.

We found the superior cornea to be the thickest part at baseline in both eyes. However, the right and left eyes did not compare with respect to the thinnest mid-peripheral corneal region at baseline with the temporal quadrant being the thinnest in the right eye and the inferior quadrant the thinnest in the left eye. The inferior mid-peripheral corneal area showed the greatest variation from baseline at most postinstillation intervals. Both the inferior and nasal midperipheral corneal areas had not returned to baseline thickness after $5 \mathrm{~min}$ with either one or two drops of oxybuprocaine HCL $0.4 \%$. As the inferior quadrant is in contact with the tear meniscus which may contain residue of the anaesthetic, there may have been a greater contact time and concentration for this quadrant. Asensio et al. ${ }^{17}$ also reported variations of greater than $10 \mu \mathrm{m}$ mostly in the inferior nasal quadrant following instillation of two drops of oxybuprocaine. The changes in corneal thickness in these areas may have implications during the course of refractive surgery as anaesthetics are often used in the pre-operative measurements. Changes in corneal thickness in the centre and $2.5 \mathrm{~mm}$ from the centre in the temporal, nasal, inferior and superior locations were assessed by Mukhopadhyyay et al. ${ }^{19}$ where topographic measurements of corneal thickness in 35 healthy right eyes were obtained using a scanning-slit device (Orbscan IIz) and a Scheimpflug device (Pentacam) before and after application of proparacaine $0.50 \%$ - sodium fluorescein $0.25 \%$. A small but significant increase of between $4.9 \mu \mathrm{m}$ and $9.1 \mu \mathrm{m}$ was recorded in all locations.

A limitation of our study is that corneal thickness changes were not measured in only the right eye or only the left eye to allow direct comparison of the effect of one drop to two drops of oxybuprocaine in the same eye. Also, the oxybuprocaine instillation was not accompanied by fluorescein which is often the combination used when performing GAT. However, Mukhopadhyay et al. ${ }^{19}$ investigating the effect on corneal thickness following instillation of a combination of $0.5 \%$ proparacaine and $0.25 \%$ sodium fluorescein on the central and mid-peripheral cornea using ultrasound pachymetry found insignificant changes to corneal thickness with the combination.

\section{Conclusion}

The results show that there are small changes to central and MPCT following the instillation of oxybuprocaine. There appears to be greater variation of mean thickness in the midperipheral cornea. These changes (especially after about $30 \mathrm{~s}$ post-instillation), however, were not found to be statistically or clinically significant, and confirm findings of other studies. Clinicians should also be aware that even though the mean change in corneal thickness was often insignificant, individual responses vary and should be considered. It is expected that corneal anaesthesia is achieved $15-20 \mathrm{~s}$ after instillation ${ }^{14,15}$ or at least after $60 \mathrm{~s},{ }^{24}$ however, it may be clinically advisable to wait at least $5 \mathrm{~min}$ before any procedure that is dependent on corneal thickness is performed. This would allow sufficient time for the corneal thickness to stabilise around baseline to avoid any discrepancies due to short-term anaestheticinduced variations on corneal thickness. Future studies on the effect of local anaesthetics on corneal thickness should 
include individuals with less pigmented irises, ocular pathology, high refractive errors, hyperopes and those who have undergone refractive surgery.

\section{Acknowledgements}

We are grateful for the contribution of SBK Nurmohamed, R Khan and T Zikhali towards this research.

\section{Competing interests}

The authors declare that they have no financial or personal relationships which may have inappropriately influenced them in writing this article.

\section{Authors' contributions}

R.G., Z.Y.S., A.R., R.H. and N.M.G. were all involved in the conceptualisation, data collection and write up of the research study.

\section{References}

1. Stone J, Phillips AJ. Contact lenses. England: Butterworths; 1984.

2. Wessels IF, Oh Y. Tonometer utilization, accuracy and calibration under field conditions. Arch Ophthalmol. 1990;108(12):1709-1712. http://dx.doi.org/10.1001/ archopht.1990.01070140063030

3. Whitacre MM, Stein R. Sources of error with use of Goldmann-type tonometers Ophthalmology. 1993;38(1):1-30. http://dx.doi.org/10.1016/0039-6257(93)90053-a

4. Ehlers N, Brausen T, Sperling S. cited in: Akram A, Yaqub A, Dar AJ, Fiaz. Pitfalls in intraocular pressure measurement by Goldmann-type applanation tonometers. Pak J Ophthalmol. 2009;25:112-118.

5. Mark HH. Corneal curvature in applanation tonometry. Am J Ophthalmol. 1973;76(2):223-224. http://dx.doi.org/10.1016/0002-9394(73)90164-5

6. Grant WM. Fluorescein for applanation tonometry, more convenient and uniform application. Am J Ophthalmol. 1963;55:1252-1253. http://dx.doi.org/10.1016/0002 9394(63)90199-5

7. Goldman H, Schmidt T. Uber applanation tonometry. Ophthalmology. 1961;141: 441-446.

8. Brusini P, Salvetat ML, Zeppieri M, Tosomi C, Parisi L. Comparison of ICare tonometer with Goldmann Applanation tonometer in glaucoma patients. J Glaucoma. 2006;15(3):213-217. http://dx.doi.org/10.1097/01.ijg.0000212208.87523.66
9. Al-Mezaine HS, Al-Obeidan S, Kangave D, Sadaawy A, Wehaib TA, Al-Amro SA. The relationship between central corneal thickness and degree of myopia among Saudi adults. Int Ophthalmol. 2009;29(5):373-378. http://dx.doi.org/10.1007/ S10792-008-9249-8

10. Huang JY, Pekmezci M, Yaplee S, Lin S. Intra-examiner repeatability and agreement of corneal pachymetry map measurement by time-domain and Fourier-domain optical coherence Huang tomography. Graef Arch Clin Exp Ophthalmol. 2010;248(11):1647-1656. http://dx.doi.org/10.1007/s00417-010-1360-7

11. Damji KF, Muni RH, Munger RM. Influence of corneal variables on accuracy of intraocular pressure measurement. J Glaucoma. 2003;12(1):69-80. http://dx.doi. org/10.1097/00061198-200302000-00015

12. Herndon LW, Choudhri SA, Cox T. Central corneal thickness in normal, glaucomatous, and ocular hypertensive eyes. Arch Ophthalmol. 1997;115(9):1137-1141. http:// dx.doi.org/10.1001/archopht.1997.01100160307007

13. Nam SM, Lee HK, Kim EK, Seo KY. Comparison of corneal thickness after the instillation of topical anaesthetics: Proparacaine versus oxybuprocaine. Cornea. 2006;25(1):51-54. http://dx.doi.org/10.1097/01.ico.0000179929.97651.59

14. Rosa N, De Bernardo M, Borrelli M, Filosa ML, Lanza M. Effect of oxybuprocaine eye drops on corneal volume and thickness measurements. Optom Vis Sci. 2011;88(5):640-644. http://dx.doi.org/10.1097/OPX.0b013e3182114303

15. Ogbuehi KC, Chijuka JC, Osuagwu UL. Changes in central corneal thickness values after instillation of oxybuprocaine hydrochloride $0.4 \%$. Cont Lens Anterior Eye. 2012;35(5):199-202. http://dx.doi.org/10.1016/j.clae.2012.05.004

16. Herse P, Siu A. Short-term effects of proparacaine on human corneal thickness. Acta Ophthalmol. 1992;70(6):740-744. http://dx.doi.org/10.1111/j.1755-3768.1992. tb04879.x

17. Asensio I, Rahhal S, Alonso L, Palanca-Sanfrancisco J, Sanchis-Gimeno J. Corneal thickness values before and after oxybuprocaine $0.4 \%$ eye drops. Cornea. 2003;22(6):527-532. http://dx.doi.org/10.1097/00003226-200308000-00008

18. Lam AK, Chen D. Effect of proparacaine on central corneal thickness values. An evaluation using noncontact specular microscopy and Pentacam. Cornea. 2007;26(1):55-58. http://dx.doi.org/10.1097/01.ico.0000240082.08416.22

19. Mukhopadhyay DR, North RV, Hamilton-Maxwell KE. Effect of proparacaine $0.50 \%$ sodium fluorescein $0.25 \% \mathrm{mix}$ and contact ultrasound pachymetry on central and mid-peripheral corneal thickness measured by noncontact optical pachymetry. Cataract Refract Surg. 2011;37(5):907-913. http://dx.doi.org/10.1016/j. jcrs.2010.11.033

20. Kim HY, Budenz DL, Lee PS, Feuer WJ, Barton K. Comparison of central corneal thickness using anterior segment optical coherence tomography vs ultrasound pachymetry. Am J Ophthalmol. 2008;145(2):228-232. http://dx.doi.org/10.1016/j. ajo.2007.09.030

21. Lázaro C, Hernández EM, Martínez D, Redondo P. Comparison of central corneal thickness measured with anterior segment optical coherence tomography versus ultrasonic pachymetry. Arch Soc Esp Oftalmol. 2013;88(2):45-49. http://dx.doi org $/ 10.1016 / \mathrm{j}$.oftal.2012.05.008

22. Muscat S, McKay N, Parks S, Kemp E, Keating D. Repeatability and reproducibility of corneal thickness measurements by optical coherence tomography. Invest Ophthalmol Vis Sci. 2002;43(6):1791-1795.

23. Penne EP, Tabbara KF. Oxubuprocaine keratopathy: A preventable disease. $\mathrm{Br}$ Ophthalmol. 1986;70(3):202-204. http://dx.doi.org/10.1136/bjo.70.3.202

24. Rosenfeld M, Logan N, Edwards KH. Optometry: Science, techniques and clinical management. China: Butterworth Heinemann Elsevier; 2009. 